\title{
Bandlimited rational models for signal reconstruction
}

\author{
K S ARUN ${ }^{1}, J_{\text {E DAVOUST }}^{2}$ and V DESAI ${ }^{3}$ \\ ${ }^{1}$ Coordinated Science Laboratory, College of Engineering, University of \\ Illinois at Urbana Champaign, 1101 West Springfield Ave., Urbana, IL \\ 61801-3082, USA \\ ${ }^{2}$ Bell Communications Research, Piscataway, NJ 08855, USA \\ ${ }^{3}$ Motorola, Arlington Heights, IL 60004, USA
}

\begin{abstract}
A rational model is proposed for reconstruction of bandlimited signals from successive observations, bandlimits, and knowledge of signal peak location. The parameters of the model may be estimated from the singular vectors of a certain double-Hankel matrix constructed from this information. In contrast to the currently popular minimum-energy approach, which has limited spectral resolution and is sensitive to noise, high spectral resolution is possible with the rational model because of its pole-zero nature. To reduce the sensitivity of the rational reconstruction to noise and modelling errors, rank reduction of the double-Hankel matrix via singular value decomposition is suggested. The singular values of the matrix provide estimates of the error in modelling the data with rational models of different orders, and so the singular value distribution can be used to select model order.
\end{abstract}

Keywords. Bandlimited extrapolation; rational models; signal reconstruction; Hankel matrix; singular value decomposition.

\section{Introduction}

A key problem encountered in the field of digital signal processing is that of reconstructing an incompletely observed discrete-index signal. This type of problem occurs in many applications, including synthetic aperture radar imaging, source bearing estimation, magnetic resonance imaging, speech processing, interferometric spectroscopy, tomagraphic imaging, and economic time series forecasting. The discrete-index signals of concern arise either from inherently discrete processes or by sampling continuous-index signals at higher than Nyquist rates. The signals in this problem have the characteristic property that they are bandlimited or almost. bandlimited. Bandlimitedness is not very restrictive since physical constraints force most signals to be bandlimited in practice.

The problem of reconstructing a bandlimited signal or image from a finite number of samples arises in many signal processing systems. The image formation problem in limited-angle tomography for instance, is one of synthesizing a two-dimensional image from a few one-dimensional projections (or line integrals of the image along 
a set of parallel lines) (Kak \& Slaney 1978; Herman 1980; Barrett \& Swindell 1981). According to the projection slice theorem (Dudgeon \& Mersereau 1984), the one-dimensional Fourier transform of each projection gives a radial slice of the two-dimensional Fourier transform of the image. Thus, the problem is equivalently one of reconstructing an image from knowledge of its Fourier transform along a few radial slices. If the image is spatially bounded, as in the tomographic reconstruction problem, its Fourier transform is a bandlimited two-dimensional sequence that is observed sparsely and needs to be extrapolated from a knowledge of its bandlimits.

A similar problem of image reconstruction from projections arises in a variety of other imaging systems including transmission electron microscopy (DeRosier \& Klug 1968), spotlight-mode synthetic aperture radar (Jenkins et al 1982), magnetic resonance imaging (Mansfield \& Morris 1982), emission computed tomography, and radio astronomical imaging (Herman 1979; Deans 1983). The bandlimited extrapolation problem appears in other guises as well in signal and image processing systems. An image restored from a blurred version might be missing information in certain frequency bands. When blurring occurs due to relative motion between camera and subject for instance, certain frequency bands are zeroed and cannot be restored. When the image to be recovered is spatially bounded, bandlimited extrapolation techniques might be used in the Fourier domain to recover the missing frequency bands.

It is well known that a continuous-index bandlimited signal, being a real analytic signal, can be perfectly extrapolated from any continuous interval by analytic continuation (Churchill \& Brown 1984) or a Taylor series expansion (Jain \& Ranganath 1981). In practice, observation noise and Taylor series truncation errors make such an extrapolation unreliable. In addition, only a finite number of discrete observations are often available in practice. Then, the uniqueness of the extrapolation is lost despite how finely the signal is sampled, how small the bandwidth is, and how large the number of observations is. Even with knowledge of the signal bandlimits, an infinite number of solutions can be found that satisfy the bandwidth constraints and match the finite number of observations. When the observations are finite in number and the spectral support (the passband) is not discrete, the bandlimited extrapolation problem always has solutions that match the data and are consistent with the bandlimits, and the solution is never unique, regardless of the number of observations and the width of the passband.

To find a unique extrapolation among the infinitely many admissible bandlimited extrapolations, some other a priori knowledge regarding the unknown signal must be utilized. Such a priori knowledge might be the energy distribution of the signal or its Fourier transform, positivity of the signal or its transform, and bounds on the signal and its spectrum. In general, incorporating such additional information narrows the set of admissible solutions at the cost of increasing the computational complexity of the extrapolation algorithm. In the absence of such a priori information, the minimum energy extrapolation is often advocated. Amongst the set of admissible solutions, it is the one with the least energy, where energy is measured by the sum of the squares $\left(l_{2}\right.$-norm). If the energy in the signal is bounded, the minimum energy extrapolation minimizes the maximum $l_{2}$ distance from every admissible solution that satisfies the bound (Potter \& Arun 1988).

The minimum-energy solution may be obtained by a variety of algorithms, some iterative (Papoulis 1975; Gerchberg 1974), and others more direct (Sabri \& Steenart 1978). It may be viewed as the result of bandpass filtering a finite-support sequence that is non-zero only at the signal observation locations. The direct algorithm 
determines the finite-support sequence from the signal observations and uses it to construct the complete signal (Jain \& Ranganath 1981). Unfortunately, the minimumenergy extrapolation is highly sensitive to noise in the observations and to errors in the passband limits. Moreover, its ability to resolve spectral peaks is limited by the size of the observation window.

Spectral resolution may be improved if the extrapolation is obtained by bandpass filtering infinite-support, finite-parameter sequences. Autoregressive and rational sequences are two examples of finite-parameter, infinite-support sequences whose parameters may be estimated from the sequences relatively easily. Experiments with these modeis have shown that while autoregressive models do a good job on spectral peaks, they fail to reproduce spectral valleys and plateaus. On the other hand, rational models can reproduce both spectral peaks and plateaus well.

In this paper, a robust extrapolation algorithm based on rational modelling is proposed when the only prior knowledge available is the location of the dominant signal peak. The Fourier transform of the signal, time-shifted so as to peak at the origin, is modelled as the ratio of two low-order polynomials in $e^{j \omega}$ (hence the term rational) within the passband and zero in the stopband. From a knowledge of the passband and the location of the signal peak, the parameters of the rational model can be estimated and used to reconstruct an admissible solution.

If prior knowledge of the region of signal energy concentration is available, it can be incorporated into the extrapolation as in Kolba \& Parks (1983) and Potter \& Arun (1989). But when only the location of the signal peak is available, and its spread is unknown, the energy concentration approach cannot be used. For such situations, this paper advocates the rational modelling approach. When the number of parameters is small, and approximate solutions to linear equations are used to estimate the parameters, this approach provides a certain degree of robustness to additive noise and bandlimit errors.

\section{The bandlimited extrapolation problem}

The discrete index set in one-dimensional problems is $Z$, the set of all integers. A $1-\mathrm{D}$, discrete-index, square summable sequence $y=\{y(n), n \in Z\}, y \in l_{2}$, is said to be bandlimited to a passband $\Omega$, a proper subset of $[-\pi, \pi]$, if its Fourier transform $Y=\mathbf{F}\{y\} \in L_{2}[-\pi, \pi]$,

$$
Y\left(e^{j \omega}\right)=\sum_{n \in Z} y(n) e^{-j \omega n}, \quad \omega \in[-\pi, \pi],
$$

is zero for all $w \notin \Omega$. Let the observation interval, a set of $p$ interger indices, be $\Delta=\left\{n_{1}, n_{2}, \ldots, n_{p}\right\}$. Define the following operators: a sampling operator $\mathbf{B}: l_{2} \rightarrow C^{p}$; a truncation operator $\mathbf{D}: L_{2}[-\pi, \pi] \rightarrow L_{2}[-\pi, \pi]$, and a bandpass operator $\mathbf{B}: l_{2} \rightarrow l_{2}$.

$$
\begin{gathered}
(\mathbf{T} y)(k)=y\left(n_{\mathbf{k}}\right), \quad k=1,2, \ldots, p, \\
(\mathbf{D} Y)\left(e^{j \omega}\right)=\left\{\begin{array}{cc}
Y\left(e^{j \omega}\right), & \text { for } \omega \in \Omega, \\
0, & \text { for } \omega \notin \Omega,
\end{array}\right. \\
\mathbf{B}=\mathbf{F}^{-1} \mathbf{D F} .
\end{gathered}
$$

In this notation, a sequence $x \in l_{2}$ is bandlimited if $\mathbf{B} x=x$. A statement of the bandlimited extrapolation problem is: 
For a given set of observations $z \in C p$, find a sequence $y \in l_{2}$ which satisfies $\mathbf{T} y=z$ and $\mathbf{B} y=y$. The problem formulation is almost identical in two or more dimensions, as well. In $m$-dimensional problems, the index set is $Z^{m}$ : the set of $m$-tuples of integers, the full frequency band is the cross product $[-\pi, \pi]^{m}$, and the $\omega n$ exponent in the Fourier transform summation is to be interpreted as the dot product between $m \times 1$ vectors $\omega$ and $n$. The rest of the problem formulation is as before.

\subsection{Minimum energy extrapolation}

The minimum energy (ME) extrapolation, which is the solution to the following probleın:

$$
\text { Minimize } \sum_{n \in Z} y(n)^{-2}, \quad \text { subject to } \mathbf{T} y=z \text { and } \mathbf{B} y=y,
$$

can be computed by iterative algorithms, such as the Papoulis-Gershberg algorithm (Papoulis 1975; Gerchberg 1974) and also by the following non-iterative two-step procedure (Sabri \& Steenart 1978)

$$
\begin{aligned}
& v=\left(\mathrm{TBT}^{*}\right)^{-1} z \\
& y=\mathrm{BT}^{*} v
\end{aligned}
$$

where $\mathbf{T}^{*}$, the adjoint of $\mathbf{T}$ simply pads zeros as

$$
\left(\mathbf{T}^{*} v\right)(n)=\left\{\begin{array}{cc}
v(k), & n=n_{k} \in \Delta, \\
0, & \text { else }
\end{array}\right.
$$

Note that the first step simply involves solving a system of $p$ linear equations, and that the composite operator TBT* is only a $p \times p$ non-singular matrix. Thus, the reconstruction algorithm is computationally simple. The minimum energy extrapolation seems to give fairly good results when the signal's energy is concentrated in a small region around the observation window (Kolba \& Parks 1983). Apart from its computational simplicity, the minimum-energy reconstruction technique has two other reasons for its popularity. When there is an energy bound on the underlying signal, the minimum-energy solution minimizes the worst-case $l_{2}$ reconstruction error (Potter \& Arun 1988). When there is an a prior estimate or nominal version of the underlying signal available, the problem of finding a reconstruction closest in $l_{2}$ norm to the nominal/prior becomes a minimum energy reconstruction problem after translation of the data by an amount that can be determined from the nominal/prior (Potter 1990). The algorithm can be easily modified to handle weighted norms (Potter 1990). The algorithm and its derivation are nearly identical in the multi-dimensional setting.

\section{Rational modelling}

The minimum energy solution can be regarded as the output of an ideal bandpass filter driven by a sequence $T^{*} v$ of finite support, as shown in figure 1 .

Thus, the spectrum of the extrapolated signal is a polynomial function of $e^{j \omega}$ in the passband

$$
\hat{Y}_{\mathrm{ME}}\left(e^{j \omega}\right)=\left\{\begin{array}{cc}
\sum_{k=0}^{p} v(k) e^{-j \omega n_{k}}, & \omega \in \Omega, \\
0, & \omega \notin \Omega,
\end{array}\right.
$$




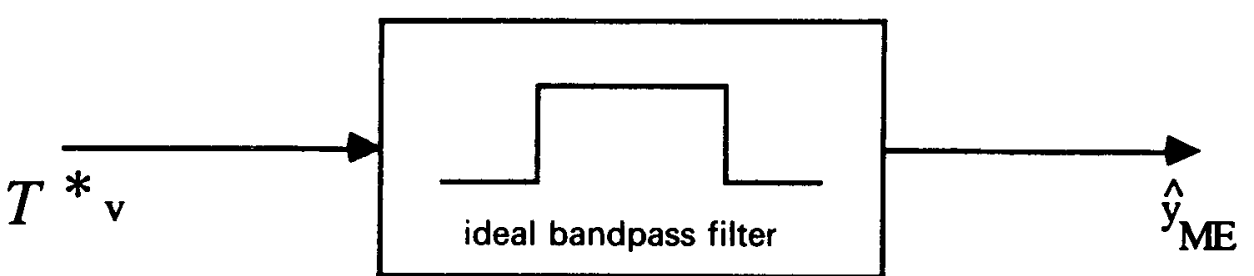

Figure 1. Interpretation of minimum energy extrapolation.

which means that the resolution ability of this spectral estimate is limited by the number of observations. There is no such resolution limit if the extrapolation is modelled as the output of the bandpass filter driven by an infinitely long sequence $h(n)$. It is obvious that every bandlimited signal can be modelled in this fashion. To be able to estimate $h(n)$ from a finite number of observations of $y, h(n)$ should admit a finite parametric representation. One such finite-parameter, infinite-support family of sequences is the rational family. Here $h(n)$ is specified by $q+1$ moving average (MA) parameters $b_{0}, b_{1}, \ldots, b_{q}$, and $p$ autoregressive (AR) parameters $a_{1}, a_{2}, \ldots a_{p}$ :

$$
h(n)=\sum_{k=1}^{p} a_{k} h(n-k)+\sum_{k=0}^{q} b_{k} \delta(n-k)
$$

where $\delta(\cdot)$ is the unit pulse sequence. The Fourier transform of $h(\cdot)$ is the ratio of a degree- $q$ polynomial to a degree- $p$ polynomial,

$$
H\left(e^{j \omega}\right)=\sum_{k=0}^{q} b_{k} e^{-j \omega k} /\left(1-\sum_{k=1}^{p} a_{k} e^{-j \omega k}\right) .
$$

The maximum of degrees $p$ and $q$ is referred to as the rational model order. Because convolution commutes, an alternate depiction of the rational model is as in figure 3. The bandlimited signal obtained from such a model satisfies the difference equation

$$
y(n)=\sum_{k=1}^{p} a_{k} y(n-k)+\sum_{k=0}^{q} b_{k} u(n-k)
$$

where $u(\cdot)$ is the impulse response of the ideal bandpass filter. In 1-D, $u(\cdot)$ is a linear combination of discrete sinc sequences. The Fourier transform of the output signal of the rational model is

$$
Y\left(e^{j \omega}\right)=\left\{\begin{array}{cc}
H\left(e^{j \omega}\right), & \omega \in \Omega \\
0, & \text { else. }
\end{array}\right.
$$

Note that $h(n)$ itself can never be bandlimited since the maximum number of zeros in $H(z)$ is $q$, whereas a bandlimited signal has an uncountable number of zeroes. Hence, the bandpass filter in the model is essential. The polynomials in the rational

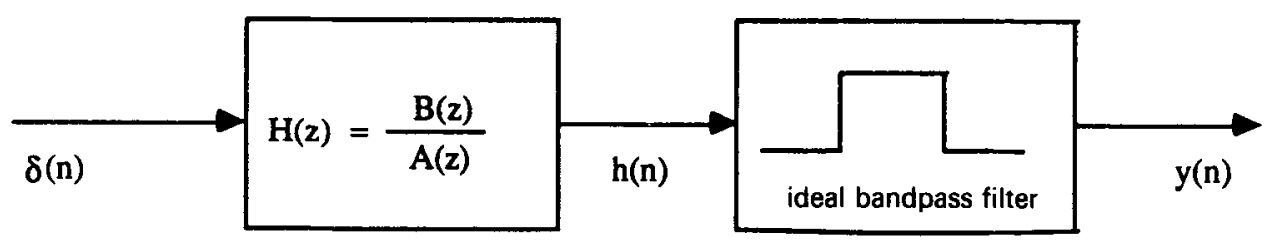

Figure 2. The rational model for $y$. 
$\underset{\substack{\text { impulse response of } \\ \text { ideal BPF }}}{\mathrm{u}(\mathrm{n})} \mathrm{H(z)}=\frac{\mathrm{B}(\mathrm{z})}{\mathrm{A}(\mathrm{z})} \underset{\mathrm{y}(\mathrm{n})}{\longrightarrow}$

Figure 3. The rational model redrawn.

transfer function can be factored as

$$
H(z)=b_{0}\left[\prod_{i=1}^{q}\left(1-\alpha_{i} z^{-1}\right) / \prod_{i=1}^{p}\left(1-\beta_{i} z^{-1}\right)\right],
$$

where $\alpha_{i}$ are zeroes and $\beta_{i}$ are poles of the transfer function. Note that if $\beta_{i}$ are close to the unit circle, then for $\omega$ near angle $\left(\beta_{i}\right), H\left(e^{j \omega}\right)$ is large, and the sharpness of the peak grows with the proximity of the pole to the unit circle. It is this behaviour of the spectrum around poles that gives the rational model its spectral resolution ability.

Another property that is noteworthy about the rational model: $y$ exhibits its strongest peak around the origin. This has nothing to do with causality of the model, but has everything to do with (implicitly assumed) stability of the model. There is no causality restriction on the model, which means that poles can be both inside and outside the unit circle. Whatever the pole locations, the implicit assumption that the Fourier transform $H\left(e^{j \omega}\right)$ exists means we are also assuming that the model is externally stable, therefore, that $h(n)$, the inverse Fourier transform of $H\left(e^{j \omega}\right)$ decays geometrically in both directions away from the time origin. The peak of $h(n)$ lies at or close to the origin, in a region whose length is determined by $p$ and $q$.

Once the model parameters are found, the reconstruction of $y(\cdot)$ is obtained in our algorithm by the inverse discrete Fourier transform (DFT) of equispaced samples of $Y\left(e^{j \omega}\right)$, which yields an approximation to the convolution of the infinitely long sequences $h$ and $u$. Since the $u(\cdot)$ sequence also has a dominant peak at 0 , the output $y$ of the convolution of $h$ and $u$ will also peak around the origin.

Thus, prior information of signal peak location has to be used to shift the time frame to make the underlying signal peak at the origin, before rational models are used.

\section{Model estimation}

In the AR model, the numerator polynomial of $H(z)$ is the constant $b_{0}$. Though not as general as the rational pole-zero model, the all-pole AR model has high spectral resolution ability, and its parameters are easily estimated (Davoust 1988). On the other hand, the MA model, for which the denominator of $H(z)$ is 1 , has limited applications due to its poor spectral resolution ability.

\subsection{The moving average (MA) model}

The $(q+1)$-parameter difference equation of the order- $q$ MA model is

$$
y(n)=\sum_{k=0}^{q} b_{k} u(n-k)
$$


and the model's transfer function is

$$
H\left(e^{j \omega}\right)=\sum_{k=0}^{q} b_{k} e^{-j \omega k}
$$

Because $H\left(e^{j \omega}\right)$ is a polynomial in $e^{j \omega}$, the MA model cannot reproduce sharp spectral peaks unless the model order is large. Thus, in the neighbourhood of several spectral peaks, this model may blend two or more peaks into a single peak. Although the MA model has poor resolution ability, it can locate zeroes and produce smooth spectral estimates.

The MA model parameters may be obtained by finding a total least-squares solution (Golub \& Van Loan 1983) of the following linear system of equations. Note that the Topelitz coefficient matrix has full rank (Kung 1978).

$$
\left(\begin{array}{cccc}
u(i+q) & u(i+q-1) & \cdots & u(i) \\
u(i+q+1) & u(i+q) & \cdots & u(i+1) \\
\cdots & \cdots & & \cdots \\
u(i+N-1) & u(i+N-2) & \cdots & u(i+N-q-1)
\end{array}\right)\left(\begin{array}{c}
b_{0} \\
b_{1} \\
\cdots \\
b_{q}
\end{array}\right)=\left(\begin{array}{c}
y(i) \\
y(i+1) \\
\cdots \\
y(i+N-1)
\end{array}\right)
$$

Since the number of model parameters can vary from 1 to $N$, the selection of model order is also a critical issue. Choosing the maximum order of $N-1$ may give the highest resolution; however, spurious peaks can be introduced into the spectral estimate. Also, when the maximum order is selected, no noise suppression occurs because the coefficient matrix is square.

An interesting property of the moving average approach is its similarity to the minimum energy approach. For both approaches, the coefficient matrix is composed only of the impulse response of the ideal bandpass filter. When the model order equals $N-1$ and the observations are at consecutive integers starting at the origin, then the minimum energy and MA approaches are identical. Thus, the MA approach is a generalization of the minimum energy approach which can use fewer parameters to achieve a certain amount of robustness to noise.

\subsection{The autoregressive (AR) model}

The $(p+1)$-parameter equation of the order-p AR model is,

$$
y(n)-\sum_{k=1}^{p} a_{k} y(n-k)=b_{0} u(n),
$$

and the model's transfer function is

$$
H\left(e^{j \omega}\right)=b_{0} /\left(1-\sum_{k=1}^{p} a_{k} e^{-j \omega k}\right) .
$$

A system of equations can be constructed from the impulse response of the ideal bandpass filter $u(\cdot)$, the observation signal $y(\cdot)$, the number of observations $N$, and the initial index $i$ of the observations, whose solution will yield the model parameters.

$$
\left(\begin{array}{ccccc}
y(i+p-1) & y(i+p-2) & \cdots & y(i) & u(i+p) \\
y(i+p) & y(i+p-1) & \cdots & y(i+1) & u(i+p+1) \\
\cdots & \cdots & & \cdots & \cdots \\
y(i+N-2) & y(i+N-3) & \cdots & y(i+N-p-1) & u(i+N-1)
\end{array}\right)\left(\begin{array}{c}
a_{1} \\
a_{2} \\
\cdots \\
a_{p} \\
b_{0}
\end{array}\right)=
$$




$$
=\left(\begin{array}{c}
y(i+p) \\
y(i+p+1) \\
\cdots \\
y(i+N-1)
\end{array}\right)
$$

By redefining the parameters, it is possible to rewrite the above so that the coefficient matrix is Toeplitz

$$
\left(\begin{array}{cccc}
y(i+p) & y(i+p-1) & \cdots & y(i) \\
y(i+p+1) & y(i+p) & \cdots & y(i+1) \\
\cdots & \cdots & & \cdots \\
y(i+N-1) & y(i+N-2) & \cdots & y(i+N-p-1)
\end{array}\right)\left(\begin{array}{c}
c_{0} \\
c_{1} \\
\cdots \\
c_{p}
\end{array}\right)=\left(\begin{array}{c}
u(i+p) \\
u(i+p+1) \\
\cdots \\
u(i+N-1)
\end{array}\right)
$$

where $c_{0}=1 / b_{0}$ and $c_{i}=-a_{i} / b_{0}, i=1,2, \ldots, p$. Usually, the model order $p$ is unknown in many applications, and it must satisfy

$$
p \leqslant\left\lfloor\frac{N-1}{2}\right\rfloor \text {. }
$$

With an odd number of observations and order $p$ equal to $(N-1) / 2$, the coefficient Toeplitz matrix becomes square and the computationally efficient Trench algorithm (Golub \& Van Loan 1983) can be employed to solve the above system. For any other model order, the parameters may be obtained by finding a total least-squares solution to the overdetermined linear system of equations.

Model order selection is a critical problem with the AR approach. Several issues need to be considered. For small orders, the coefficient matrix is overdetermined and the deleterious effects of noise are reduced by finding a total least-squares solution. The square coefficient matrix, which results from selecting the maximum order, does not allow for averaging out the effects of additive noise. Moreover, overmodelling introduces spurious peaks in the spectral estimate. Alternatively, choosing a small model order may cause low resolution in the spectral estimate. A good rule of thumb is to select a model order that is approximately a third of the number of observations.

\subsection{The general pole-zero or ARMA model}

The general pole-zero model combines the properties of the AR and MA models and is the most versatile, doing a good job both at peaks and at valleys. To determine the parameters of the rational model from the observations of $y$, construct the following double-Hankel matrix A

$$
\mathbf{A}=\left(\begin{array}{c}
\mathbf{Y} p+1 \\
\mathbf{U} q+1
\end{array}\right)
$$

where $\mathbf{Y} p+1$ and $\mathbf{U} q+1$ are defined below as matrices with $p+1$ rows and $q+1$ rows, respectively, and as many $N-p+1$ columns, where $N$ is the number of observations, $p$ and $q$ are the model orders, and $i$ is the initial index of the observation (signal peak is assumed to be at zero, without loss of generality).

$$
\mathbf{Y} p+1=\left(\begin{array}{cccc}
y(i) & y(i+1) & \cdots & y(i+N-p) \\
y(i+1) & y(i+2) & \cdots & y(i+N-p+1) \\
\cdots & \cdots & & \cdots \\
y(i+p) & y(i+p+1) & \cdots & y(i+N)
\end{array}\right)
$$


and

$$
\mathbf{U} q+1=\left(\begin{array}{cccc}
u(i) & u(i+1) & \cdots & u(i+N-p) \\
u(i+1) & u(i+2) & \cdots & u(i+N-p+1) \\
\cdots & \cdots & & \cdots \\
u(i+q) & u(i+q+1) & \cdots & u(i+N-p+q)
\end{array}\right)
$$

If $N \geqslant 2 p+q+1$, then $n=\left(a_{p} a_{p-1} \ldots a_{1}-1 b_{q} b_{q-1} \ldots b_{0}\right)^{t}$ is a null vector of $\mathbf{A}^{t}$. Here the superscript $t$ denotes transposition of the matrix or vector. Thus, the model parameters may be obtained by setting up a linear system of equations using $y$ and $u$, and solving for the parameters. To fit such a model approximately to the observations, the system can solved by using total least squares (Golub \& Van Loan 1983). Then, the solution is the appropriately scaled left singular vector of $\mathbf{A}$ corresponding to the smallest singular value.

Order selection is once again a problem. It has to be done before parameter estimation in the above scheme. Qualitative prior information about the signal may be used to pick $p$ and $q$. For instance, a rational model with a large denominator order should be selected for impulsive spectra. Similarly, modelling a smooth spectrum requires large numerator order.

The general rational model has many desirable properties; the major drawback is difficulty in choosing the model order. The next section addresses this limitation and suggests methods for determining model order from the observations directly.

Though in setting up the equations for the MA, AR, and ARMA models, it was assumed that the observation indices in $\Delta$ were consecutive integers, the assumption is not needed. Similar equations can be set up in all three cases, even when $\Delta$ is taken as the union of multiple segments of consecutive integers, as long as each segment is at least $[\max (p, q)+1]$-long (Davoust 1988). A potentially more useful generalization is in multidimensional reconstruction problems. If, as before, $\left\{u(n), n \in Z^{m}\right\}$ is the $m$-dimensional impulse response of the ideal bandpass filter for passband $\Omega$ (a proper subset of $\left.[-\pi, \pi]^{m}\right)$, the rational model is

$$
y(n)=\sum_{k \in N_{a}} a_{k} y(n-k)+\sum_{k \in N_{b}} b_{k} u(n-k),
$$

where $N_{a}$ and $N_{b}$ are $m-\mathrm{D}$ masks and $k$ and $n$ are $m$-tuples of integers from $Z^{m}$. The recursion is illustrated for a specific example in 2-D in figure 4. Mask sizes have to be below a certain limit for model parameters to be identifiable from given data. Given observation region $\Delta$ from $Z^{m}$, let $R$ be the set of indices in $Z^{m}$ which satisfy the following condition: When the masks $N_{a}$ and $N_{b}$ are centred at an index in $R$, all indices covered by the masks must lie in $\Delta$. Mathematically,

$$
R=\left\{n \in Z^{m}: n-k \in \Delta \text { for all } k \in N_{a} \text { or } N_{b}\right\} .
$$

For some examples of 2-D masks and their regions $R$, see figure 5 . A system of linear equations, one for each index in $R$, can be set up to solve for the model parameters (Davoust 1988). Thus, the system is square or overdetermined only if the size of $R$ is greater than $p+q=$ size $N_{a}+$ size $N_{b}$. When the system is overdetermined, the parameters are estimated by total least squares.

\section{A useful rank property}

The parametric estimation scheme introduced in the previous section requires that the model order be known in advance. Next we show how to determine the model 


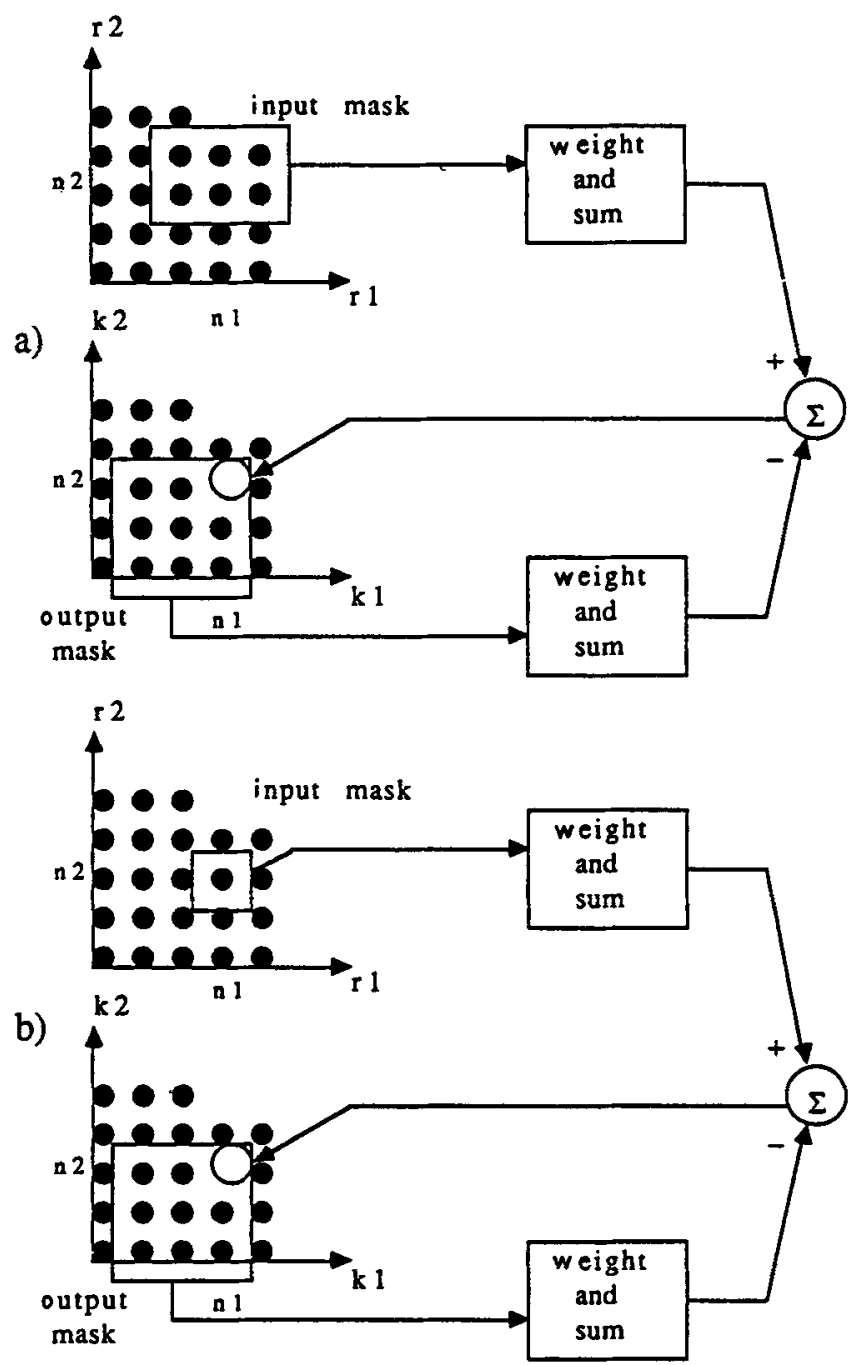

Figure 4. Schematic representation of 2-D rational and AR systems. (a) General 2-D rational system. (b) Simplified all-pole system.

order in the 1-D case from the rank of a double-Hankel matrix and show how to obtain the model parameters from a factorization of the matrix using singular value decomposition (SVD).

\section{PROPOSITION}

Given $N$ consecutive observations starting at index $i$ of a bandlimited signal $y(\cdot)$, the bandlimits of its passband $\Omega$, the following double-Hankel matrix $A$ with $2 \kappa$ rows and $N-\kappa+2$ columns may be constructed,

$$
\mathbf{A}=\left(\begin{array}{l}
\mathbf{Y}_{\boldsymbol{\kappa}} \\
\mathbf{U} \boldsymbol{\kappa}
\end{array}\right),
$$

where $\kappa=\llcorner(N+1) / 3\lrcorner$, and $u(\cdot)$ is the impulse response of the ideal bandpass filter whose passband is $\Omega$. If $y$ admits a $(p, q)$-order rational model, then matrix $\mathbf{A}$ has 
Some 2-D filter masks:

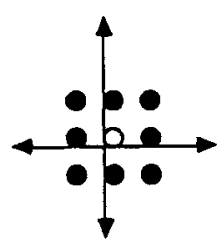

(a) square noncausal

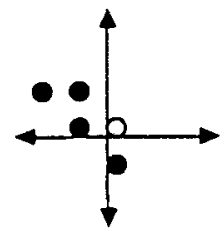

(c) irregular

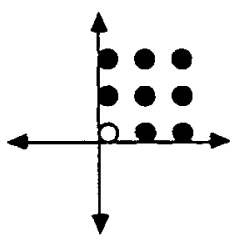

(b) square causal

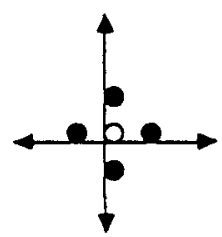

(d) noncausal

and the regions $\mathcal{R}$ they define:

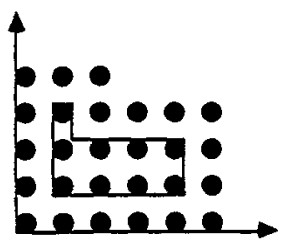

(a)

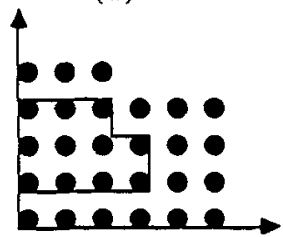

(c)

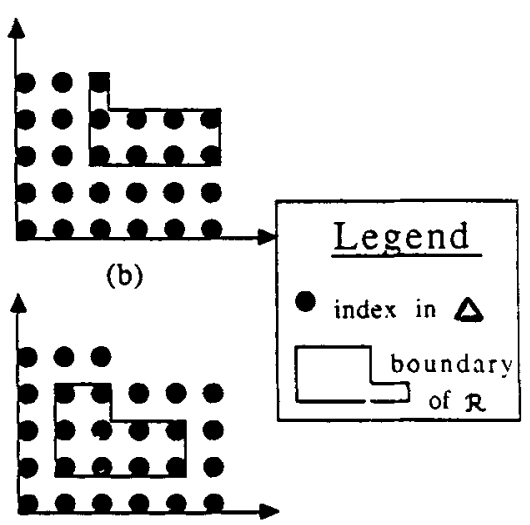

(d)

Figure 5. Some choices of 2-D masks and their corresponding regions $R$.

rank defect, provided $\kappa>\max (p, q)=\mu$,

$$
\operatorname{rank}(\mathbf{A})=\kappa+\max (p, q)=\kappa+\mu .
$$

Proof. Because of the difference equation relating $y$ and $u, \mathbf{A}$ has the following $\kappa-\mu$ linearly independent left null vectors, i.e. $n_{i}^{t} \mathrm{~A}=0^{t}$ :

$$
\begin{gathered}
n_{1}^{t}=\left(a_{\mu}, a_{\mu-1}, \ldots a_{1},-1,0,0, \ldots 0,0, b_{\mu}, b_{\mu-1}, \ldots b_{1}, b_{0}, 0,0, \ldots 0,0\right), \\
n_{2}^{t}=\left(0, a_{\mu}, \ldots a_{2}, a_{1},-1,0, \ldots 0,0, b_{\mu}, b_{\mu-1}, \ldots b_{2}, b_{1}, b_{0}, 0, \ldots 0,0\right), \\
\ldots \\
n_{\kappa-\mu}^{t}=\left(0,0, \ldots 0,0, a_{\mu}, a_{\mu-1}, \ldots a_{1},-1,0,0, \ldots 0,0, b_{\mu}, b_{\mu-1}, \ldots b_{1}, b_{0}\right) .
\end{gathered}
$$

It can be shown that if $\mathbf{A}$ has other null vectors that are linearly independent of the above vectors, then $y$ admits a rational model of order less than $\mu$ over the observation interval. Thus, if $\mu$ is the minimal order needed to model the observations, then

$$
\operatorname{rank}(\mathrm{A})=2 \kappa-(\kappa-\mu)=\kappa+\mu .
$$

Hence the proposition. 
Note that the number $\kappa$ is picked to make $\mathbf{A}$ either square or as close to being square as possible. A more general version of this proposition was shown by DeMoor and coworkers in the context of system identification (De Moor et al 1988).

When fitting the rational model to a set of given data and bandlimits, the matrix might turn out to be full rank. Then, SVD can be used to estimate modelling errors for different model orders and to pick an acceptable order. Once the model order is known, either the signal space or the noise space can be employed to find the model parameters. Signal-space methods use the orthogonality between the parameter vector and the principal singular vectors. If $u_{1}, u_{2}, \ldots, u_{\kappa+\mu}$ are the left singular vectors corresponding to the largest $k+\cdot m$ singular values, then we expect

$$
\left(\begin{array}{c}
u_{1}^{t} \\
u_{2}^{t} \\
\ldots \\
u_{\kappa+\mu}^{t}
\end{array}\right)\left(n_{1} n_{2} \ldots n_{\kappa-\mu}\right) \approx 0
$$

This leads to a $(\kappa-\mu)(\kappa+\mu) \times(2 \mu+1)$ linear system of equations which can be solved using total least squares for the model parameters $a_{i}$ and $b_{i}, i=1,2, \ldots, \mu$.

\subsection{A low rank factorization}

State space parameters of the rational model in non-canonical realizations can also be found from the SVD of $\mathbf{A}$. Consider the following state space representation for the rational model of figure 3 .

$$
\begin{aligned}
& x(j+1)=\mathbf{A} x(j)+b u(j), \\
& y(j)=c^{\imath} x(j)+h(0) u(j) .
\end{aligned}
$$

The rational transfer function is related to the state space parameters as

$$
H(z)=c^{t}(z I-\mathbf{A})^{-1} b+h(0) .
$$

It can also be seen that

$$
y(j)=c^{s} \mathbf{A}^{j-i} x(i)+\sum_{m=1}^{j-i} c^{t} \mathbf{A}^{m-1} b u(j-m)+h(0) u(j), \quad j \geqslant i,
$$

which can be written in matrix notation using the extended observability matrix $\Theta$ and the Markov parameter matrix $\mathbf{L}$, as

where

$$
\mathbf{Y} \kappa=\mathbf{L U} \kappa+\boldsymbol{\Theta X}
$$

and

$$
\mathbf{L}=\left(\begin{array}{cccc}
h(0) & 0 & \cdots & 0 \\
c^{t} b & h(0) & \cdots & 0 \\
c^{t} A b & c^{t} b & \cdots & 0 \\
\cdots & \cdots & . & \cdots \\
c^{t} \mathbf{A}^{\kappa-3} b & c^{t} \mathbf{A}^{\kappa-4} b & \cdots & 0 \\
c^{t} \mathbf{A}^{\kappa-2} b & c^{t} \mathbf{A}^{\alpha-3} b & \cdots & h(0)
\end{array}\right), \quad \Theta=\left(\begin{array}{c}
c^{t} \\
c^{t} \mathbf{A} \\
c^{t} \mathbf{A}^{2} \\
\cdots \\
c^{t} \mathbf{A}^{\kappa-1}
\end{array}\right)
$$

$$
\mathbf{X}=(x(\mathbf{i}) x(i+1) \ldots x(i+N-\kappa)) \text {. }
$$


This leads to the following factorization of the double-Hankel matrix,

$$
\mathbf{A}=\left(\begin{array}{c}
\mathbf{Y}_{\boldsymbol{\kappa}} \\
\mathbf{U}_{\boldsymbol{\kappa}}
\end{array}\right)=\left(\begin{array}{ll}
\mathbf{L} & \boldsymbol{\Theta} \\
\mathbf{I} & \mathbf{0}
\end{array}\right)\left(\begin{array}{c}
\mathbf{U}_{\boldsymbol{\kappa}} \\
\mathbf{X}
\end{array}\right)
$$

with inner dimension $\kappa+\mu=\operatorname{rank}(\mathbf{A})$.

\subsection{Model estimation from singular vectors}

The above factorization shows that information about $\mathbf{L}$ and $\boldsymbol{\theta}$ is available in the left singular vectors of the double-Hankel matrix $A$. If the SVD of $A$ is

$$
\mathbf{A}=U \Sigma V^{t}=\left(u_{1} u_{2} \ldots u_{2 \kappa}\right)\left(\begin{array}{cccc}
\sigma_{1} & 0 & \cdots & 0 \\
0 & \sigma_{2} & \cdots & 0 \\
\vdots & \vdots & \cdots & \vdots \\
0 & 0 & \cdots & \sigma_{2 \kappa}
\end{array}\right)\left(\begin{array}{c}
v_{1}^{t} \\
v_{2}^{t} \\
\cdots \\
v_{2 \kappa}^{t}
\end{array}\right)=\sum_{i=1}^{2 \kappa} \sigma_{i} u_{i} v_{i}^{t}
$$

with singular values indexed in decreasing order of magnitude, then, provided that the $\mu$-order model is appropriate, $\sigma_{\kappa+\mu+1}$ and smaller singular values will be significantly smaller than the largest $\kappa+\mu$ singular value. Therefore,

$$
\sum_{i=1}^{\kappa+\mu} \sigma_{i} u_{i} v_{i}^{t}=\left(u_{1} u_{2} \ldots u_{\kappa+\mu}\right)\left(\begin{array}{cccc}
\sigma_{1} & 0 & \cdots & 0 \\
0 & \sigma_{2} & \cdots & 0 \\
\vdots & \vdots & \cdots & \vdots \\
0 & 0 & \cdots & \sigma_{\kappa+\mu}
\end{array}\right)\left(\begin{array}{c}
v_{1}^{t} \\
v_{2}^{t} \\
\cdots \\
v_{\kappa+\mu}^{t}
\end{array}\right)=U_{1} \Sigma_{1} V_{1}^{t}
$$

is approximately equal to

$$
\left(\begin{array}{ll}
\mathbf{L} & \boldsymbol{\Theta} \\
\mathbf{I} & \mathbf{0}
\end{array}\right) T^{-1} T\left(\begin{array}{c}
\mathbf{U} \kappa \\
\mathbf{X}
\end{array}\right),
$$

where $T$ is any invertible $(\kappa+\mu) \times(\kappa+\mu)$ transformation. The following identification is then possible,

$$
\left(\begin{array}{cc}
\mathbf{L} & \boldsymbol{\Theta} \\
\mathbf{I} & \mathbf{0}
\end{array}\right)=U_{1} \Sigma_{1}^{1 / 2} .
$$

Partition $U_{1} \Sigma_{1}^{1 / 2}$ as $\left(\begin{array}{c}P_{1} P_{2} \\ P_{3} P_{4}\end{array}\right)$, where $P_{1}$ and $P_{3}$ are $\kappa \times \kappa$ matrices, and $P_{2}$ and $P_{4}$ are $\kappa \times \mu$ matrices, then perform a $Q R$ decomposition of $\left(P_{3} P_{4}\right)^{t}$ as

$$
\left(P_{3} P_{4}\right) Q=\left(R^{t} 0\right)
$$

where $Q$ is unitary and $R$ is $\kappa \times \kappa$ upper triangular. Then,

$$
\begin{aligned}
\mathbf{L}+\Theta F_{1} & =P_{1} Q R^{t}, \\
\Theta F_{2} & =P_{2} Q R^{t},
\end{aligned}
$$

where $F_{1}$ and $F_{2}$ are a pair of unknown matrices relating to the model. Recognizing 
that similarity transformations change the state space parameters as

$$
\left(A, b, c^{t}\right) \rightarrow\left(S^{-1} A S, S^{-1} b, c^{t} S\right), \quad \Theta \rightarrow \Theta S
$$

we estimate the model parameters in a new coordinate system where

$$
\begin{aligned}
& \Theta=P_{2} Q R^{t} \\
& \mathbf{L}+\Theta F_{1}^{-1} F_{2}=P_{1} Q R^{t} .
\end{aligned}
$$

The observability matrix $\Theta$ thus found, can be used to find $A$ and $c$ as in Kung (1978). Matrix $\Theta_{1}$ (likewise $\Theta_{2}$ ) is obtained from $\Theta$ by deleting the last row (likewise the first row) and $A$ is obtained as the total least squares solution of

$$
\Theta_{1} A=\Theta_{2} \text {. }
$$

The output matrix $c^{t}$ is identified as the first row of $\Theta$. The input matrix $b$ may be found by exploiting the lower triangular structure of $L$ (Desai 1990).

\section{Example reconstructions}

The following representative computer experiments on synthetic data serve to demonstrate the potential of the rational modelling approach.

Experiment 1: In the first simulation experiment, the test signal has the following discontinuous Fourier transform:

$$
Y\left(e^{j \omega}\right)=\left\{\begin{array}{l}
3,0-2 \pi \leqslant|\omega| \leqslant 0 \cdot 25 \pi \text { radians, } \\
5,0 \cdot 35 \pi \leqslant|\omega| \leqslant 0 \cdot 4 \pi \text { radians, } \\
0, \text { otherwise. }
\end{array}\right.
$$

This is a difficult spectrum to model with a rational model, because of the sharp discontinuities and the flat areas. The given data consists of twenty observations beginning at index 8 . The passband limits of $0.2 \pi$ and $0.4 \pi$ radians are given, along

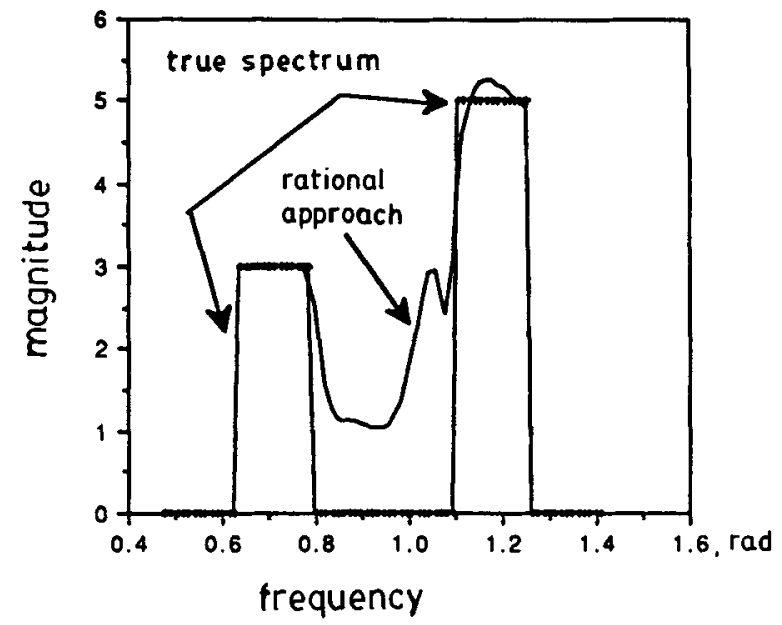

Figure 6. Sixth order rational estimate of the 1-D spectrum of experiment 1. 


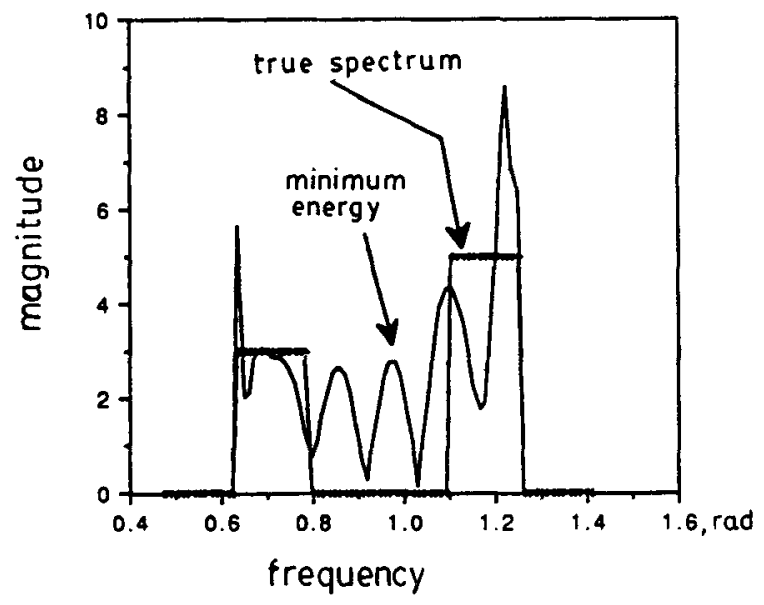

Figure 7. Minimum energy estimate of the spectrum of experiment 1 .

with the information that the signal peaks at 0 . The reconstruction obtained from a sixth-order rational (pole-zero) model is depicted in figure 6. It shows that the salient features of the spectrum were reproduced. The one spurious peak in the passband is due to a pole being placed at $\omega=0.35 \pi$ radians, the discontinuity in the spectrum. In general, the rational modelling approach places poles where the true spectrum has abrupt changes and discontinuities. The minimum energy estimate in figure 7 poorly reproduces the spectrum and also has many more spurious peaks in the spectral estimate. The ninth order AR estimate is shown in figure 8. The sixth order rational estimate in the presence of $100 \mathrm{~dB}$ noise is shown in figure 9.

Experiment 2: The test signal in the second experiment is two-dimensional and also has two dominant peaks in the spectrum. As shown in figure 10, the spectrum has two rectangular peaks of height 21 centred at

$$
\left(\omega_{1}=0, \omega_{2}=0.45\right) \text { and at }\left(\omega_{1}=-0.45, \omega_{2}=-0.45\right) \text { radians }
$$

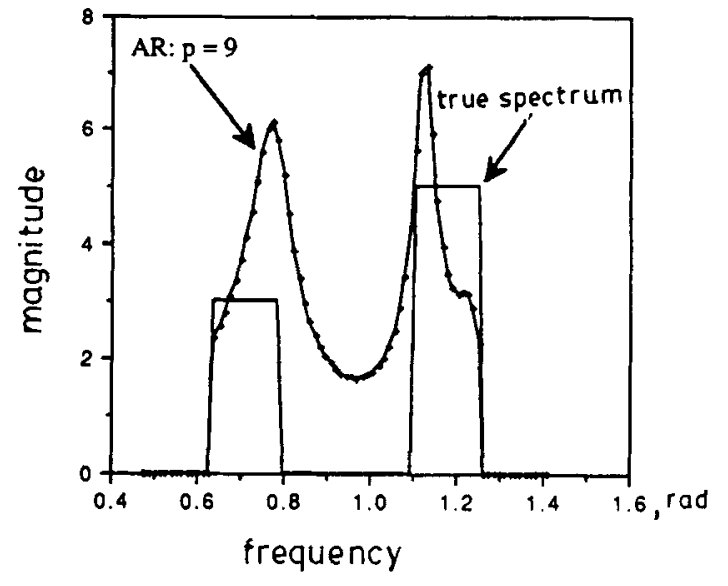

Figure 8. Ninth order AR estimate of the spectrum of experiment 1 . 


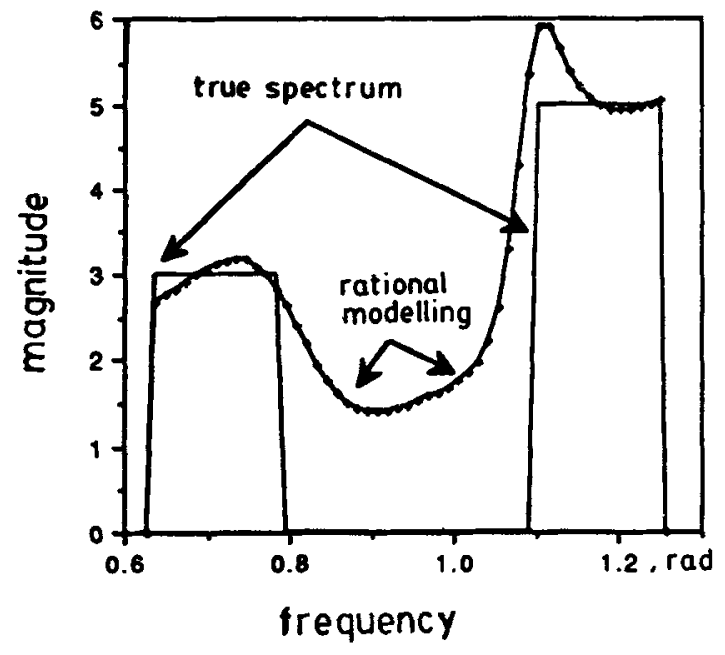

Figure 9. Rational reconstruction of the spectrum of experiment 1 in the presence of $100 \mathrm{~dB}$ noise.

situated on top of a circular pedestal of height 4 and radius $\pi / 3$ radians. All 2-D mesh and contour plots in figures 10-12 are drawn by sampling the spectra on a $64 \times 64$ grid. The observations are in the signal domain, centred around the peak at the origin on a $13 \times 13$ square. Given these observations and the passband region as the circte of radius $\pi / 3$, AR reconstruction was attempted using a square $7 \times 7$ casual mask. Figure 11 shows that the AR reconstruction locates the two peaks and the base well.

Experiment 3: The AR algorithm is also effective in direction finding of far-field point sources with uniform, wide-band sensor arrays, when gross bounds on the source temporal spectrum band and direction are available. The spatio-temporal signal received in the sensor array from a single source is given by the expression

$$
x(m, n)=\frac{\sin ((k m+n) \delta) \cos \left((k m+n) \omega_{c}\right)}{\pi^{2}(k n+m)}
$$

where $\omega_{c}$ is the centre frequency of the source's temporal spectrum, $\delta$ is related to the bandwidth source temporal spectrum, and $k$ is related to the direction of arrival of the plane wave from the source. The ideal spectrum of this signal is a 2-D impulse sheet along a line of slope $k$, passing through the origin. The support of the impulse sheet is restricted to a region of length $2 \delta\left(1+k^{2}\right)^{1 / 2}$ along this line. In our experiment, $\omega_{c}=1, k=0.5$, and $\delta=0.4$. Observations were from a $9 \times 9$ square region in spatio-temporal $(m, n)$ domain, centred at the origin. The data were corrupted by white Gaussian noise, at $0 \mathrm{~dB}$ SNR. The following overestimate of the passband was given: two rectangular regions delimited by $\left|\omega_{1}\right|<1.0$ radians and $0.5<\left|\omega_{2}\right|<1.5$ radians respectively. Figure 12 shows the AR reconstruction of the spatio-temporal spectrum using a $5 \times 5$ square mask. Notice that the reconstructed spectrum has a rational approximation to an impulse sheet, and that it can be used to estimate the slope $k$ and through it, the direction of arrival.

For more extensive simulations, the reader is referred to Davoust (1988) and Desai (1990). Briefly, our simulations indicated that small errors in signal peak location information did not affect the reconstruction significantly, but the reconstruction quality improves considerably with refined knowledge of the passband. 


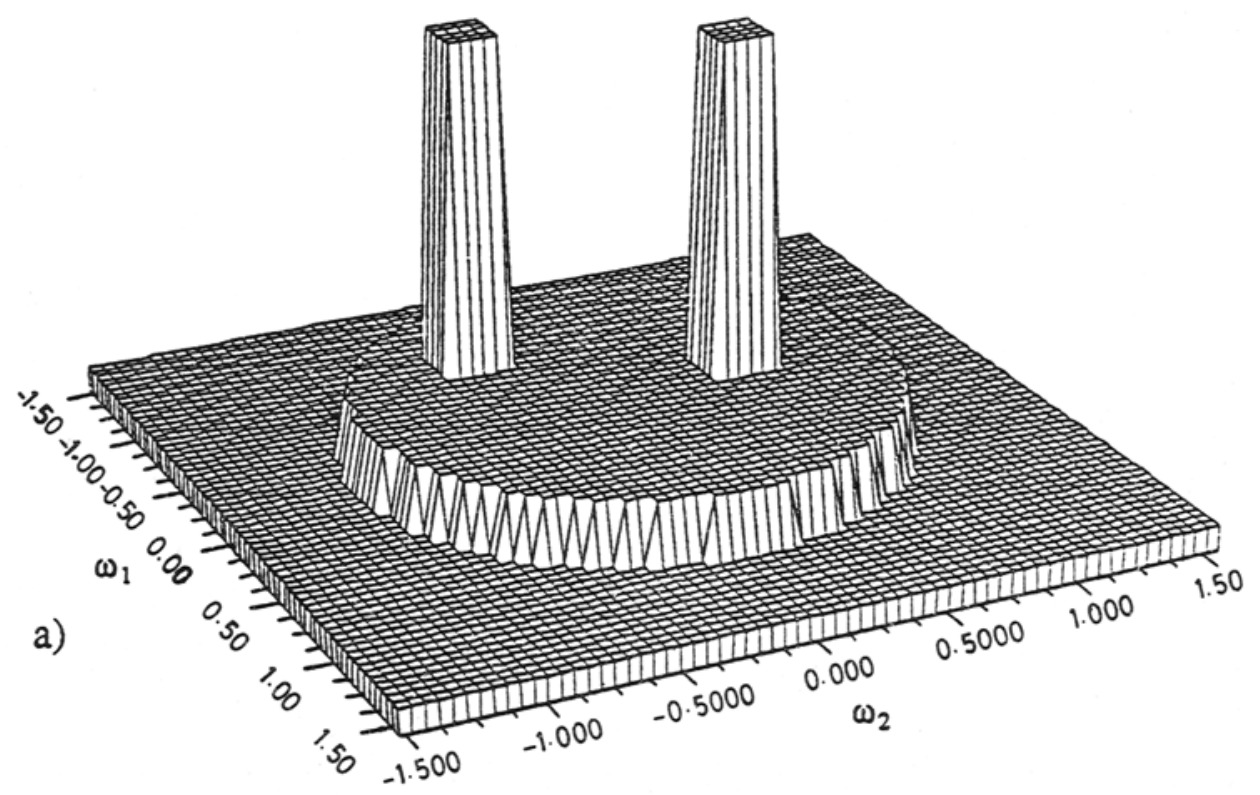

b)

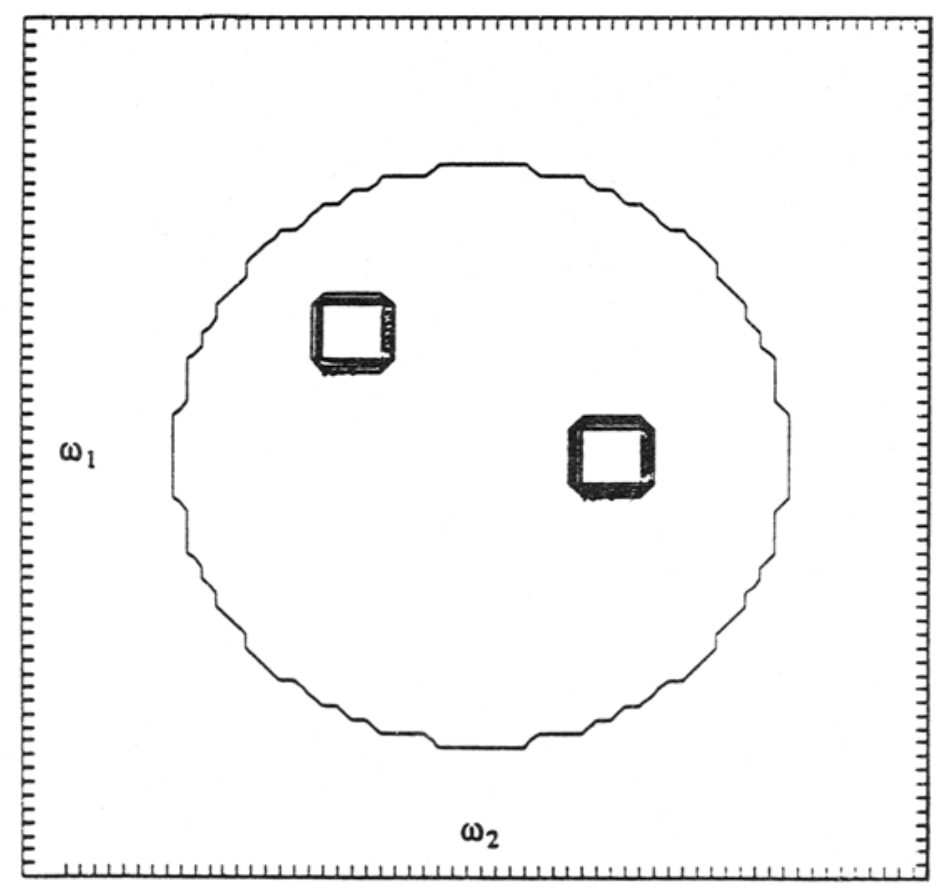

Figure 10. 2-D test spectrum of experiment 2. (a) Mesh plot, (b) contour plot. 


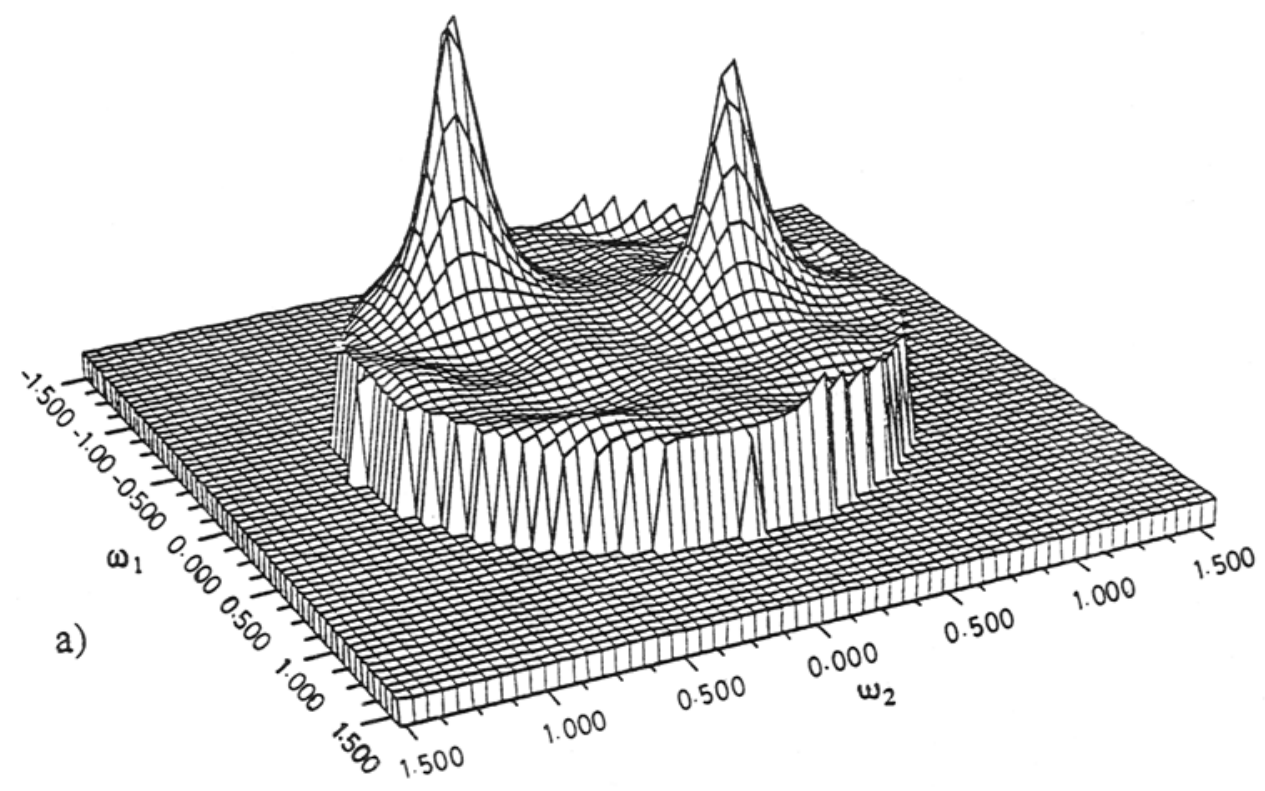

b)

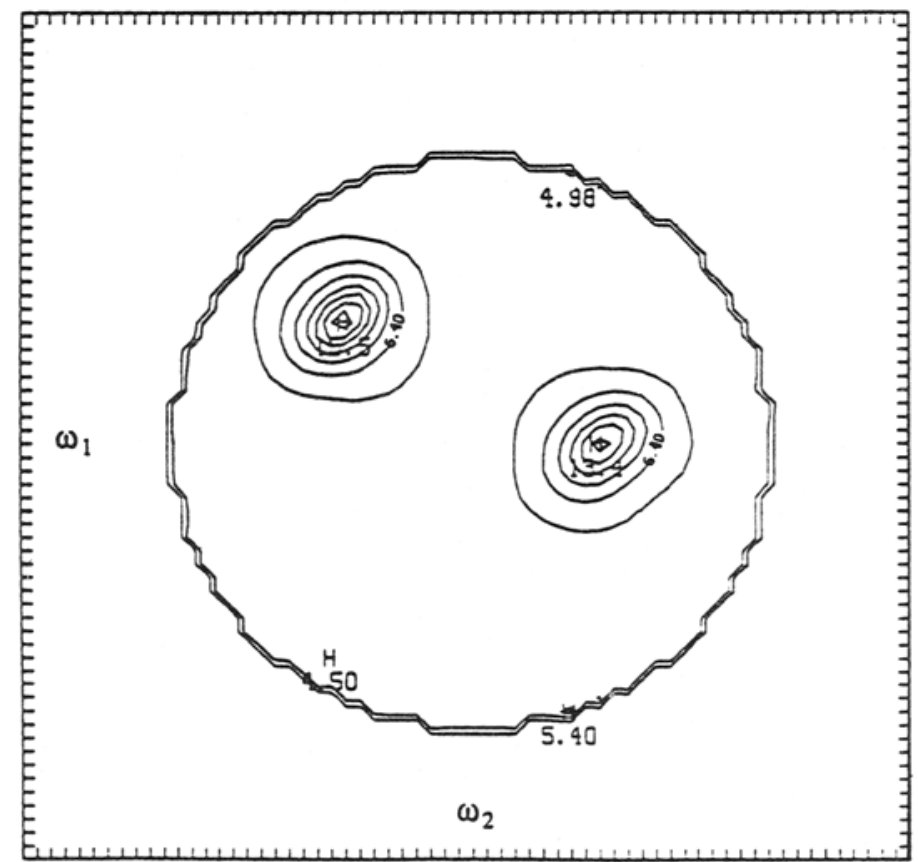

Figure 11. AR $(7 \times 7)$ reconstruction of the spectrum of experiment 2. (a) Mesh plot, (b) contour plot. 


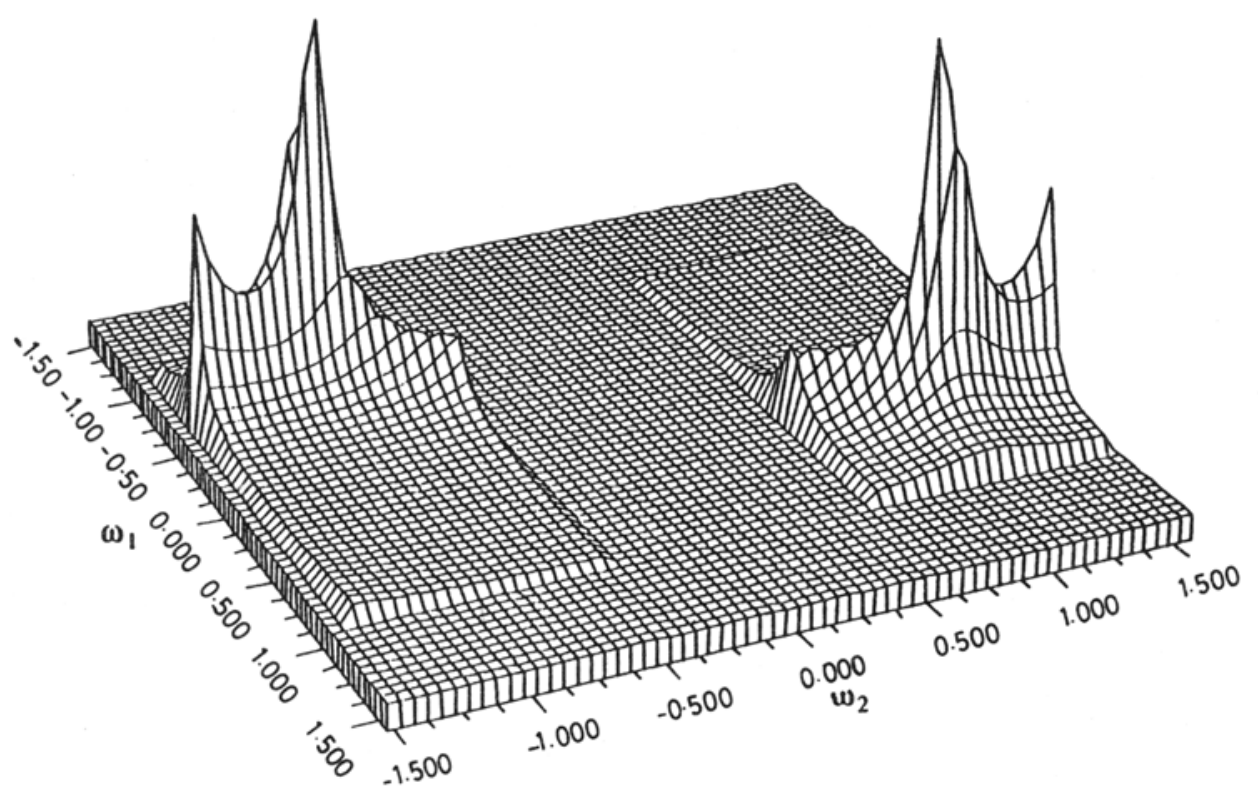

Figure 12. AR $(5 \times 5)$ reconstruction of the $2-D$ spectrum of the spatio-temporal signal of experiment 3 .

To summarize, this paper presents a rational modelling technique for bandlimited extrapolation, which can reproduce spectral peaks using few parameters, and is fairly robust to noise, to modelling errors, and to bandlimit errors.

This work was partially supported by SDIO/IST under contract DAAL 03-86-0111 administered by the US Army Research Office, by the Bell Communication Research's Graduate Study Program, and by Cray Research Inc. under grants DMS9880010N and ECS890002N and utilized the Cray X-MP/48 at the National Center for Supercomputing Applications at the University of Illinois at Urbana-Champaign.

\section{References}

Barrett H H, Swindell W 1981 Radiologic imaging: The theory of image formation (New York: Academic Press) vol 2

Churchill R V, Brown J W 1984 Complex variables and applications (New York: McGraw-Hill)

Davoust J 1988 AR bandlimited extrapolation of multidimensional signals, M S thesis, Department of Electrical and Computer Engineering, University of Illinois, Urbana

Deans S R 1983 The Radon transform and its applications (New York: Wiley Interscience)

De Moor B, Moonen M, Vaadenberghe L, Vandewalle K 1988 Identification of linear state space models with singular value decomposition using canonical correlation concepts. SVD and signal processing (ed.) E F Deprettere (Amsterdam: Elsevier Science)

DeRosier D J. Klug A 1968 Reconstruction of three-dimensional structures from electron micrographs. Nature (London) 217: 130

Desai V 1990 Robust extrapolation of bandlimited signals by rational modelling, $\mathrm{M} S$ thesis, Department of Electrical and Computer Engineering, University of Illinois, Urbana

Dudgeon D E, Mersereau R M 1984 Multi-dimensional digital signal processing (Englewood Cliffs, NJ: Prentice Hall) 
Gerchberg R W 1974 Super resolution through error energy reduction. Opt. Acta 21: 709-720

Golub G H, Van Loan C F 1983 Matrix computations (Baltimore: John Hopkins University Press)

Herman G T 1979 Image reconstruction from projections: Implementation and applications. Topies in Applied Physics Series (Berlin: Springer Verlag) vol. 32

Herman G T 1980 Image reconstruction from projections: The fundamentals of computerized tomography (San Francisco: Academic Press)

Jain A K, Ranganath S 1981 Extrapolation alogrithms for discrete signal with application in spectral estimation. IEEE Trans. Acoust. Speech. Signal Process. ASSP-29: 830-845

Jenkins W K, Munson D C Jr, O'Brien J D 1982 Commonalities in the theory of signal processing for synthetic aperture radar, computer-aided tomography, and beam-forming sonar. Proc. 25th Midwest Symposium on Circuits and Systems (New York: IEEE Press) pp 175-178

Kak A C, Slaney M 1978 Principles of computerized tomographic imaging (New York: IEEE Press)

Kolba D P, Parks T W 1983 Optimum estimation for bandlimited signals including time domain considerations. IEEE Trans. Acoust. Speech. Signal Process. ASSP-31: 113-122

Kung S Y 1978 A new identification and model reduction algorithm via singular value decomposition. Proc. Twelfth Annular Asilomar Conference on Circuits, Systems, and Computers (New York: IEEE Press) pp 705-714

Mansfield P, Morris P G 1882 NMR imaging in biomedicine (New York: Academic Press)

Papoulis A 1975 A new algorithm in spectral analysis and bandlimited extrapolation. IEEE Trans. Circuits Syst. CAS-22: 735-742

Potter L C 1990 Constrained signal reconstruction, Ph D dissertation, Dept. of Electrical and Computer Engineering, University of Illinois, Urbana

Potter L C, Arun K S 1988 An iterative algorithm for minimum-norm extrapolation. Proc. 22nd Annu. Asilomar Conf. on Signals, Systems, and Computers (New York: IEEE Press)

Potter L. C, Arun K S 1989 Energy concentration in bandlimited extrapolation. IEEE Trans. Acoust. Speech, Signal Process. ASSP-37: 1027-1041

Sabri M S, Steenart W 1978 An approach to bandlimited signal extrapolation: The extrapolation matrix. IEEE Trans. Circuits Syst. CAS-25: 74-78 